\title{
INTEGRATED BLIND ELECTRONIC EQUALIZER FOR FIBER DISPERSION COMPENSATION
}

\author{
Foster F. Dai, Senior Member, IEEE, Shengfang Wei, Richard Jaeger, Fellow, IEEE \\ Electrical and Computer Engineering Dept., 200 Broun Hall, Auburn University, Auburn, AL 36849-5201 \\ Tel. 334-844-1863, Fax. 334-844-1809, Email: Daifa01@eng.auburn.edu
}

\begin{abstract}
This paper presents an adaptive blind electronic equalization technique for fiber dispersion compensation. Constant-modulus blind adaptive algorithm is proposed to provide reference-free feedback. The proposed $10 \mathrm{~Gb} / \mathrm{s} 7$-tap electronic equalizer is designed in a $50 \mathrm{GHz} \mathrm{SiGe}$ technology and the blind feed back algorithm can be implemented in an FPGA with $100 \mathrm{MHz}$ operation frequency.
\end{abstract}

Keywords: fiber dispersion, chromatic dispersion, PMD, blind equalization, CMA

\section{INTRODUCTION}

Modal, chromatic and polarization mode dispersions are the major sources of transmission impairments in high data rate fiber communications. Without proper compensation, the performance of the fiber communication systems will be severely limited. The available dispersion compensation fiber is static in nature, therefore does not support agile optical networks. Other optical solutions are only capable of compensating one form of the dispersions with very high cost, high insertion loss and slow tuning speed if they are tunable at all. This paper presents an adaptive electronic equalizer for the compensation of all forms of fiber dispersions, including modal, chromatic and polarization mode dispersions. The electronic equalizer can be dynamically tuned at high speed and has much smaller form factor and much lower cost. An electronic equalizer can be integrated into a single chip using the high-speed Silicon Germanium (SiGe) or Indium Phosphorus (InP) technology [1]. Further cost reduction is possible if electronic equalizer and other circuits on the receiver are integrated on the same die. An electronic equalizer module is superior considering its cost, size, reliability, flexibility and speed.

\section{ELECTRONIC EQUALIZERS IN ADAPTIVE FIBER RECEIVER}

Fig. 1 shows an adaptive receiver with feed forward equalizer (FFE) and decision feedback equalizer (DFE). The received optical signal is converted to electrical signal using a photo-detector (PD). Due to the square-law-detection, the amplitude of the electrical signal is proportional to the intensity of the optical signal. A trans-impedance amplifier (TIA) is used to convert the electrical current mode signal to voltage mode signal. Then, the voltage signal is fed to the data input of the feed-forward equalizer (FFE), which performs the dispersion compensation for the distorted voltage signal. The output of the FFE is experienced additional compensation at a decision feedback equalizer (DFE). The compensated signal is then fed into a clock-data recovery block (CDR) to recover the clock.

FFE, a linear equalizer, is used to compensate for moderate amplitude distortion. The equalizer is said to be linear in the sense that the estimate of a desired response is linearly related to the input vector when steady state conditions are established. On the other hand, a decision feedback equalizer is used to improve receiver performance in the presence of moderate to severe amplitude distortion. A DFE is intrinsically nonlinear as it feeds back to the input a sequence of hard decisions made at the equalizer output. Herein lie both the strength and weakness of the DFE. The feedback section removes the lagging inter-symbol interference that is beyond the reach of the forward section. However, the DFE suffers from the problem of error propagation since hard decisions may destroy information: once a wrong decision is made, errors are fed back to the equalizer input, which in turn leads to a higher probability of error on subsequent decisions. The end result could be a burst of errors. Moreover, like any feedback system, a DFE design involves stability analysis, while a FFE is always stable.

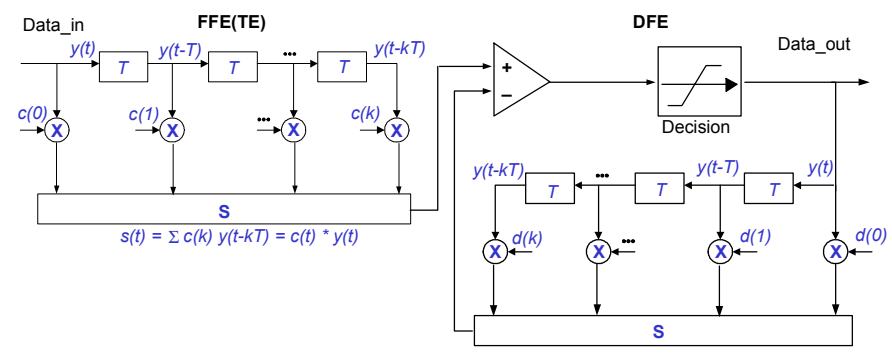

Fig.1 Block diagram of an electronic equalizer including TE and DFE.

3. ADAPTIVE EQUALIZER FEEDBACK ALGORITHM 
Various adaptive equalizer algorithms have been developed for digital signal processing. LMS maximum likelihood algorithms are widely used in equalizer designs. Recursive least-squares (RLS) algorithm achieves faster convergence, but is computationally more complex than LMS since matrix inversion is required. The reference signal required by the LMS algorithm can be either provided by a transmitted training sequence or estimated by the detected bits. Decisiondirected tap adaptation uses the output of the slicer/decision circuit as an estimation of the transmitted sequence. LMS is simple and can be implemented for FFE and DFE at $10 \mathrm{~Gb} / \mathrm{s}$ data rate. For higher data rate, steepest descend algorithm can be used, which consecutively dithers the equalizer taps to achieve the optimized signal quality. As a simplified variant of LMS, sign-sign LMS algorithm evaluates only the signal signs, which has greatly reduced computation time, yet with very "noisy" gradient estimate. The biggest advantages of sign-sign LMS algorithm is that it does not require any highspeed ADC and thus can be implemented at high speed.

The drawback of conventional equalization algorithms lies on the fact that they need the assistance of a training sequence, which is not suitable for applications with varying channel environment. On the other hand, blind equalization provides a reference-free equalization algorithm, which meets the current fiber communication transmission standard. Blind equalization is thus critical for agile optical networks, where the channel environment varies constantly. Since binary ONOFF key modulation with constant envelope is used in fiber communication system, constant-modulus adaptive algorithm (CMA) is proposed for fiber dispersion compensation using blind equalization.

\subsection{LMS equalizer with training sequence}

If a reference training sequence is transmitted during a preassigned time slot, the equalizer coefficients can be adapted by using LMS adaptive algorithm so that the output of the equalizer closely matches the training sequence. The LMS reference can also be estimated by the detected bits. In this case, a blind equalization is implemented with the goal of widening the eye-open for improved BER. If $r_{k}$ is the reference signal and $y_{k}$ is the equalizer output at time instance $k$, respectively, the error signal of LMS is defined as

$$
e_{k}=r_{k}-y_{k}=r_{k}-X_{k}^{T} W_{k}
$$

where $r_{k}$ is the reference (training) signal, $y_{k}$ is the equalizer output at time instance $k$. The cost function to be minimized is defined as

$$
J_{k}=e_{k}^{2}=\left[r_{k}-X_{k}^{T} W_{k}\right]^{2}
$$

In each iteration of the adaptive process, we have a gradient estimate of

$$
\nabla J_{k}=\frac{\partial J_{k}}{\partial W_{k}}=2 e_{k} \frac{\partial e_{k}}{\partial W_{k}}=-2 e_{k} X_{k}
$$

With this simple estimate of the gradient we can specify a steepest descent adaptive algorithm that is described by the following equations

$$
W_{k+1}=W_{k}-\mu \nabla J_{k}=W_{k}+2 \mu e_{k} X_{k}
$$

\subsection{CMA blind equalizer}

Inclusion of the training sequence with the transmitted information adds an overhead and thus reduces the throughput of the system. Therefore, to reduce the system overhead, adaptation schemes are preferred that do not require training. In blind equalization, instead of using the training sequence, one or more properties of the transmitted signal are used to estimate the inverse of the dispersive channel. Unlike LMS that assumes a reference $r_{k}$, the error $e_{k}$ in Eq. (1) cannot be used to define the cost function in blind equalization. Since the transmitted signal in fiber communication is of the constant amplitude, we can assume constant amplitude $A$ in the absence of signal degradations. Then, the error signal in blind equalization can be defined as [3]

$$
e_{k}=\left|y_{k}\right|^{2}-A^{2}
$$

Further define the cost function as

$$
J_{k}=\mathrm{E}\left\{e_{k}^{2}\right\}=\mathrm{E}\left\{\left(y_{k}^{2}-A^{2}\right)^{2}\right\}
$$

The true gradient of $J$ at time $k$ could be approximated by its instantaneous value

$$
\nabla J_{k}=\frac{\partial J_{k}}{\partial W_{k}}=2 e_{k} \frac{\partial e_{k}}{\partial W_{k}}=4 e_{k} y_{k} X_{k}=4\left(y_{k}^{2}-A^{2}\right) y_{k} X_{k}
$$

And the CMA recursion expression becomes

$$
W_{k+1}=W_{k}-\mu \nabla J_{k}=W_{k}-\mu\left(y_{k}^{2}-A^{2}\right) y_{k} X_{k}
$$

where $y_{k}=W_{k}^{\mathrm{T} *} X_{k}$

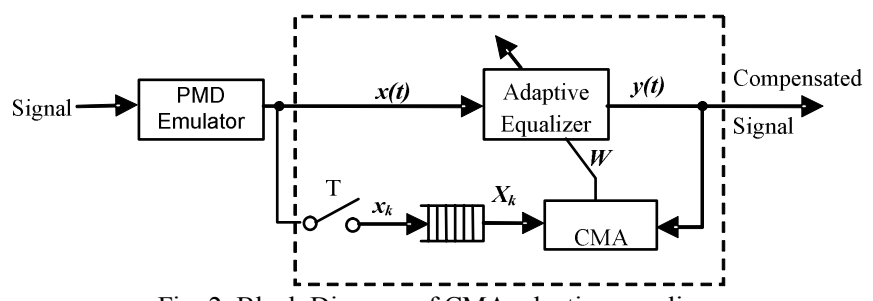

Fig. 2. Block Diagram of CMA adaptive equalizer

\section{SIMULATION RESULTS}

\subsection{LMS equalizer}

To demonstrate the effect of electronic equalizer on fiber dispersion compensation, we have conducted system level simulations in MATLAB. We simulated a 7-tap analog feed forward equalizer (FFE) with LMS feedback algorithm for PMD compensation (Fig. 3) and chromatic dispersion compensation (Fig. 4). Note that the severely distorted signal can be compensated using just 7 taps in an analog transversal equalizer. With the adaptive feedback, the tap coefficients are constantly adjusted based on the error signal. Even without feedback, the fixed coefficient equalizer can still open the distorted eye. For fixed network, we can thus predetermine the equalizer tap coefficients and load them into the equalizer at the field, which is very attractive from a cost reduction point of view. For chromatic dispersion, tap coefficients can be programmed based on the length and type of the fiber, providing a much more economic way than the current solution using dispersion compensation fiber (DCF), which is static and also wavelength dependent. 


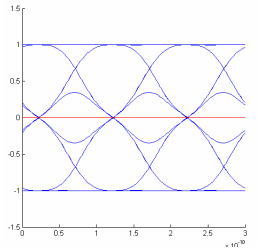

(a)

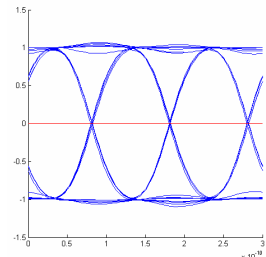

(b)

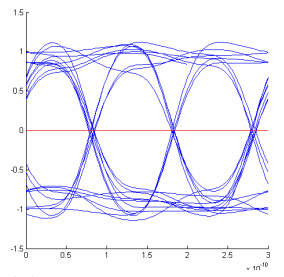

(c)
Fig.3 PMD compensation using a 7 tap transversal equalizer. (a) Eye diagram distorted by 1 st order PMD with power splitting ratio $=0.5$ and $\mathrm{DGD}=0.8$ period. (b) Compensated eye diagram with LMS adaptive feedback. (c) Compensated eye diagram with fixed tap coefficients.

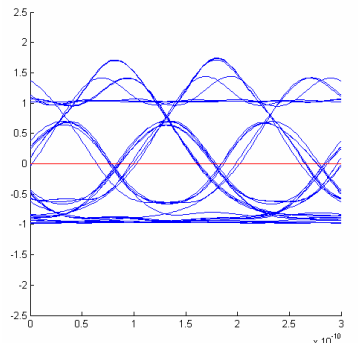

(a)

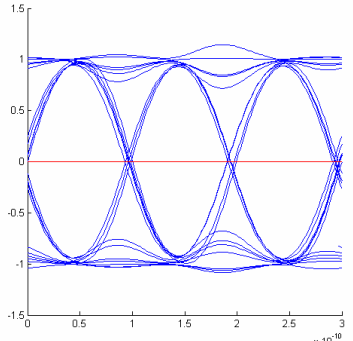

(b)
Fig. 4 Chromatic dispersion compensation using a 7 tap analog FFE equalizer. (a) Eye diagram distorted by chromatic dispersion after transmission in a $75 \mathrm{~km}$ SMF-28 fiber at wavelength $1550 \mathrm{~nm}$. (b) Compensated eye diagram with LMS adaptive feedback.

\subsection{CMA blind equalizer}

In our simulation for blind equalization, we set signal amplitude $A$ to 1 and normalized the input signal to the range of $[-1,1]$. Baud-Spaced CMA (sampling period equals to the symbol period) is used in our equalizer, and $W_{0}$ is initialized to be "Single-Spike" [2] (one non-zero tap coefficient, and all other to 0 ).

Three scenarios with different setting of DGD are investigated. Symbol period is set to be $0.025 \mathrm{~ns}(25 \mathrm{e}-12$ seconds). Simulation results when DGD is set to $48 \%$ symbol period, $64 \%$ the symbol period, and $80 \%$ the symbol period, are shown in Fig. 5, Fig. 6, and Fig. 7 respectively. In all scenarios, initial $\mathrm{W}_{0}$ is chosen to be $\left[\begin{array}{llllll}0 & 0 & 0 & 1 & 0 & 0\end{array}\right]^{\mathrm{T}}$, and feedback gain $\mu$ is set to 0.005 . Assuming the eye is constant along the horizontal direction and the eye-open is then measured as the vertical height between the mean of peak amplitudes and the mean of valley amplitudes, which converges to value 2 ideally.

From the results, we see that when DGD is less than half (48\%) symbol period (Fig. 5), the eye could be fully opened quite fast (in about $10 \mathrm{~ns}$, or 400 symbol periods); when DGD increases to $64 \%$ of symbol period (Fig. 6), the eye can be almost fully opened in less than 50ns or 2000 symbol periods; when DGD is further increased to $80 \%$ of symbol period (Fig. 7), it takes more than $100 \mathrm{~ns}$ for the coefficients to get stable, and the cost function $J$ can not converge to 0 and stays around 0.1 (Fig. 7-d), but the eye is opened much wider (Fig. 7-b) than the distorted one (Fig. 7-a).
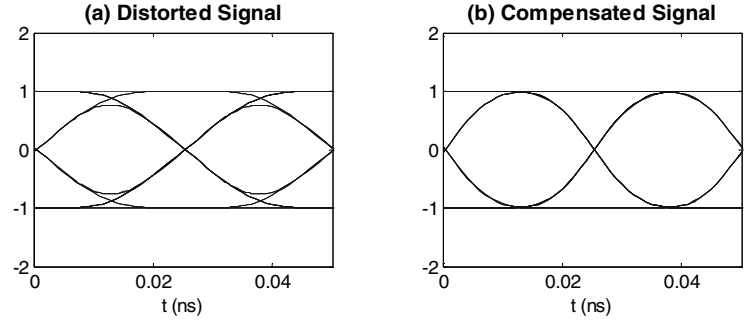

(c) Coefficien

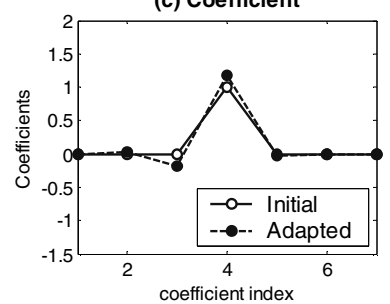

(d) Eye Opening/J

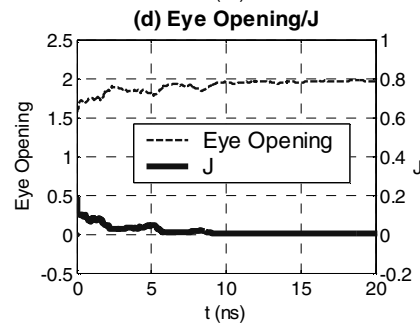

Fig. $5 \mathrm{DGD}=48 \%$ symbol period. (a), (b) Eye diagrams before/after the compensation, and they are plotted within a time window of two symbol periods and trace number of 20; (c) Initial and adapted coefficients; (d) Eye Opening and cost function $J . J$ is defined in (6) and ideally converges to 0 . (a) Distorted Signa

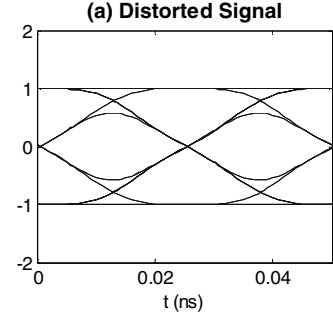

(c) Coefficient

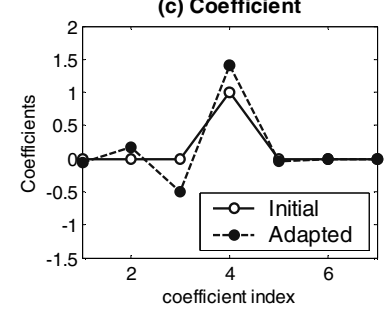

(b) Compensated Signal

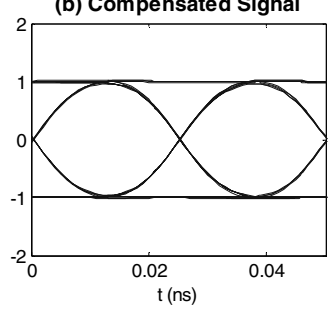

(d) Eye Opening/J

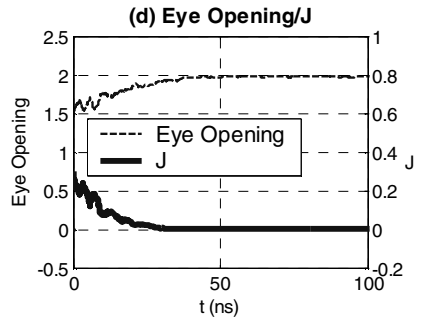

Fig. 6: $\mathrm{DGD}=64 \%$ symbol period. (Refer to the notes on Fig 5 for the meaning of each measure)

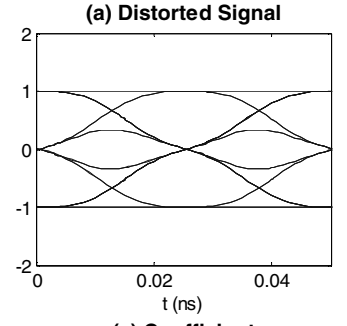

(c) Coefficient

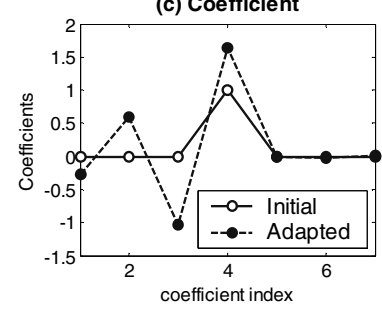

(b) Compensated Signal

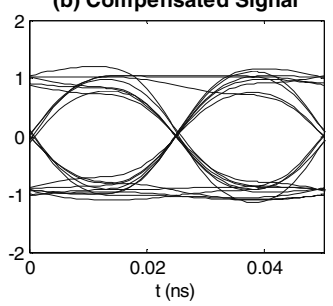

(d) Eye Opening/J

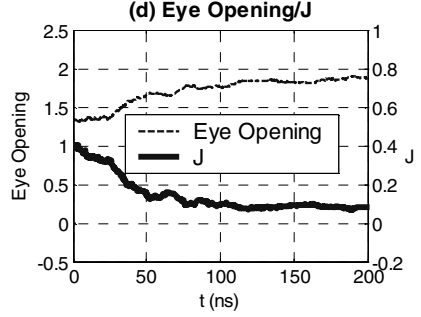

Fig. 7: DGD $=80 \%$ symbol period. (Refer to the notes on Fig 5 for the meaning of each measure) 


\section{IMPLEMENTATION OF HIGH SPEED FEED FORWARD EQUALIZER IN SIGE TECHNOLOGY}

The proposed 7-tap electronic equalizer was implemented in a $50 \mathrm{GHz} \mathrm{SiGe}$ technology. The critical block of the equalizer is the variable gain stage, which requires $10 \mathrm{GHz}$ bandwidth with small phase group delay for OC-192 fiber networks. The maximum gain of the variable gain stage is set such that its output would not saturate the following stage under any circumstance. A Gilbert gain-cell is used for this purpose and the simplified circuit is given in Fig. 8. The simulated magnitude of the equalizer tap gain under various gain settings is shown in Fig. 9, demonstrating linear gain tuning with larger than $10 \mathrm{GHz}$ bandwidth. The tap delay line is implemented using the cascaded unity gain stages with total delay of half data period (50ps). The equalizer circuit design is given in details in [5].

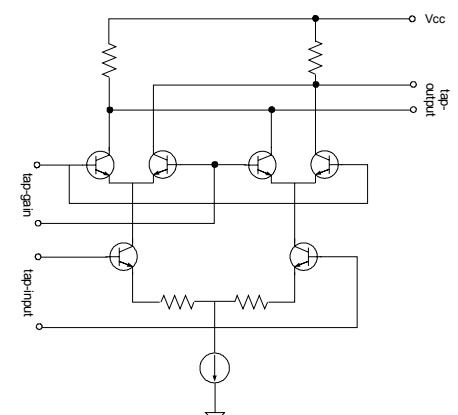

Fig. 8 Simplified equalizer tap gain stage.

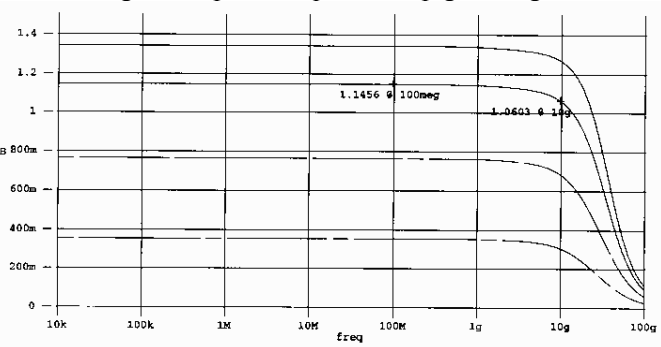

Fig.9 Magnitude of equalizer tap gain under various gain settings.

\section{IMPLEMENTATION OF CMA BLIND EQUALIZION FEEDBACK IN A 100MHZ FPGA}

The current CMA algorithm is very easy to implement. Based on (9) and (8), the implementation is shown in Fig. 10. If the filter size is $\mathrm{L}$, we see that only $\mathrm{L}$ accumulators, $3 \mathrm{~L}+1$ multipliers, and $\mathrm{L}$ adders/subtractors are needed for CMA algorithm (Fig. 10). Note that

i) The small gradient gain $\boldsymbol{\mu}$ in (8) doesn't really need a multiplier. If $\boldsymbol{\mu}$ is chosen to be $1 / 256(\approx 0.004)$, we actually only need to truncate (discard) the lowest 8 bits $(\mathrm{A}=8$ in Fig. 10).

ii) Although there are $3 \mathrm{~L}+1$ multipliers, most of them can work in parallel, for example, the left most $\mathrm{L}$ multipliers in the $1^{\text {st }}$ stage, middle $(\mathrm{L}+1)$ multipliers in the $2^{\text {nd }}$ stage, and right most $\mathrm{L}$ multipliers in the $3^{\text {rd }}$ stage, therefore the delay due to multiplier is only $3 T_{m u l}$, where $T_{m u l}$ is the average multiplication time. iii) We have different clock signals for the sampling (CLK1 in Fig. 10), and the coefficient updating (CLK2 in Fig. 10) which is much slower than CLK1. Note that multiplier has extra CLK which is not shown in the block diagram.

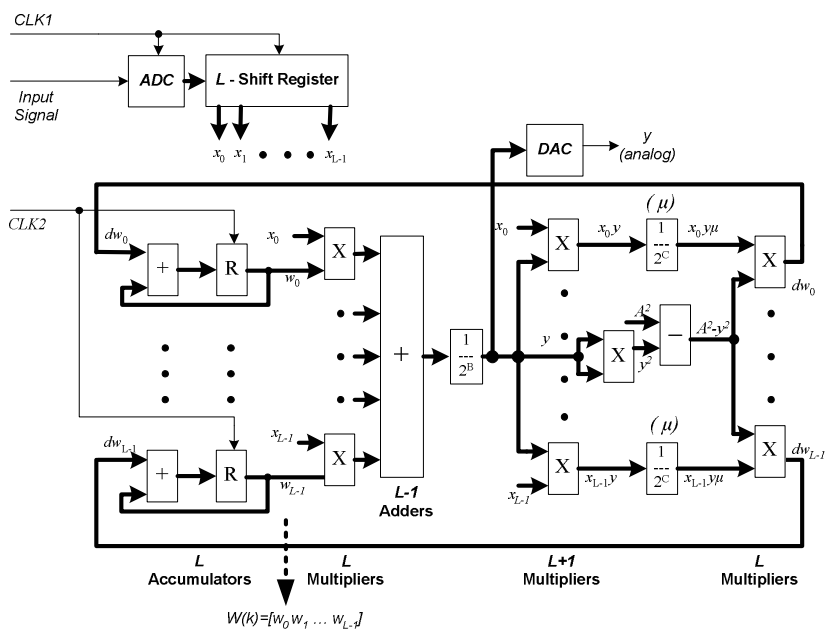

Fig. 10 Block Diagram of hardware implementation. CLK1: Sample Clock (equal to symbol rate); CLK2: Coefficient Updating Clock L: Length of Equalizer; A: Constant Amplitude $1 / 2^{\mathrm{B}}$ and $1 / 2^{\mathrm{C}}$ : bit "Truncator" (takes off the lowest $\mathrm{B} / \mathrm{C}$ bits), (where $\mathrm{C}=\left[\log _{2} \mu\right], \mathrm{B}=$ number of fractional bits in fixed point format of coefficient $\mathrm{w}$ ) $\mu$ : step size (gradient gain) Bolded lines represent multiple bits (bus) signals and thin lines for analog and control signals

\section{CONCLUSIONS}

This paper presents a novel CMA based blind electronic equalization technique for fiber dispersion compensation. Simulation demonstrated effective compensation means for chromatic and PMD dispersions using the LMS and CMA blind equation techniques.

\section{REFERENCES}

[1] Azadet, K.; Haratsch, E.F.; Kim, H.; Saibi, F.; Saunders, J.H.; Shaffer, M.; Song, L.; Meng-Lin Yu, "Equalization and FEC techniques for optical transceivers," IEEE Journal of Solid-State Circuits, Vol. 37, 317 -327, (2002).

[2] C. Richard Johnson, Jr., Philip Schniter, Thomas J. Endres, James D.Behm, Donald R. Brown, Raúl A. Casas, "Blind equalization using the Constant Modulus Criterion: a review," Proceeding of the IEEE - 86(10):1927--1950, October 1998.

[3] Michael G. Larimore, C. Richard Johnson, John R. Treichler, "Theory \& design of adaptive filters," ISBN 0130402656 , Published March 2001.

[4] J.R. Treichler and B.G. Agee, "A new approach to multipath correction of constant modulus signals," IEEE Trans. On Acoustics, Speech, and Signal Processing, vol. ASSP-31, 459-472, (1983).

[5] V. Kakani, F. F. Dai, and R. C. Jaeger, "An High Speed Integrated Equalizer for Dispersion Compensation in $10 \mathrm{~Gb} / \mathrm{s}$ Fiber Networks", IEEE International Symposium on Circuits and Systems (ISCAS), Kobe, Japan, May 2005. 